\title{
BMJ Open Third-wave cognitive therapy versus mentalisation-based treatment for major depressive disorder: a randomised clinical trial
}

\author{
Janus Christian Jakobsen, ${ }^{1,2}$ Christian Gluud, ${ }^{2}$ Mickey Kongerslev, ${ }^{1}$ \\ Kirsten Aaskov Larsen, ${ }^{3}$ Per Sørensen, ${ }^{4}$ Per Winkel, ${ }^{2}$ Theis Lange, ${ }^{5}$ \\ Ulf Søgaard, ${ }^{3}$ Erik Simonsen ${ }^{1,6}$
}

To cite: Jakobsen JC, Gluud C, Kongerslev M, et al. Third-wave cognitive therapy versus mentalisation-based treatment for major depressive disorder: a randomised clinical trial. $B M J$ Open 2014;4:e004903. doi:10.1136/bmjopen-2014004903

- Prepublication history and additional material is available. To view please visit the journal (http://dx.doi.org/ 10.1136/bmjopen-2014004903).

Received 22 January 2013 Revised 22 July 2014 Accepted 23 July 2014

CrossMark

For numbered affiliations see end of article.

\section{Correspondence to}

Dr Janus Christian Jakobsen; jcj@ctu.dk

\section{ABSTRACT}

Objective: To compare the benefits and harms of third-wave cognitive therapy versus mentalisationbased therapy in a small sample of depressed participants.

Setting: The trial was conducted at an outpatient psychiatric clinic for non-psychotic patients in Roskilde, Denmark.

Participants: 44 consecutive adult participants diagnosed with major depressive disorder.

Interventions: 18 weeks of third-wave cognitive therapy $(n=22)$ versus 18 weeks of mentalisation-based treatment $(n=22)$.

Outcomes: The primary outcome was the Hamilton Rating Scale for Depression (HDRS) at end of treatment (18 weeks). Secondary outcomes were: remission (HDRS <8), Beck's Depression Inventory, Symptom Checklist 90 Revised and The WHO-Five Well-being Index 1999.

Results: The trial inclusion lasted for about 2 years as planned but only 44 out of the planned 84 participants were randomised. Two mentalisation-based participants were lost to follow-up. The unadjusted analysis showed that third-wave participants compared with mentalisation-based participants did not differ significantly regarding the 18 weeks HDRS score (12.9 vs 17.0 ; mean difference $-4.14 ; 95 \% \mathrm{Cl}-8.30$ to $0.03 ; p=0.051$ ). In the analysis adjusted for baseline HDRS score, the difference was favouring third-wave cognitive therapy $(p=0.039)$. At 18 weeks, five of the third-wave participants $(22.7 \%)$ were in remission versus none of the mentalisation-based participants $(p=0.049)$. We recorded no suicide attempts or suicides during the intervention period in any of the 44 participants. No significant differences were found between the two intervention groups on the remaining secondary outcomes.

Conclusions: Third-wave cognitive therapy may be more effective than mentalisation-based therapy for depressive symptoms measured on the HDRS.

However, more randomised clinical trials are needed to assess the effects of third-wave cognitive therapy and mentalisation-based treatment for depression.

\section{Strengths and limitations of this study}

- It was possible to conduct the trial with a low risk of bias (adequate allocation sequence generation, adequate allocation concealment, adequate blinding, no risk of selective outcome reporting, low risk of incomplete outcome data bias, no risk of 'for profit' bias), which was the primary strength of this randomised clinical trial.

- The trial also provided valuable information about possible intervention effects of third-wave cognitive therapy and mentalisation-based treatment. Our preliminary results may be used to design future trials including estimation of sample size calculations.

- The primary limitation of this randomised clinical trial was that only 44 out of the planned 84 participants were randomised in this small-scale trial.

Trial registration number: Registered with Clinical Trials government identifier: NCT01070134.

\section{INTRODUCTION}

Third-wave cognitive therapy

Prior to this trial we carried out a systematic review of randomised clinical trials examining the effects of traditional cognitive therapy versus no intervention for major depressive disorder. ${ }^{1}$ We found that cognitive therapy compared with no intervention seems to have a small statistically significant beneficial effect on depressive symptoms. However, we identified only a limited number of relatively small randomised clinical trials all with a high risk of bias. ${ }^{1}$ During the past two decades new forms of cognitive therapy have been developed. These third-wave cognitive therapies include, for example, acceptance and commitment 
therapy, schema therapy, mindfulness-based cognitive therapy and meta-cognitive therapy. ${ }^{2}$ Especially mindfulness-based interventions have been implemented in numerous different clinical contexts in recent years. ${ }^{3-5}$ One meta-analysis showed that third-wave cognitive therapy might prevent relapse of depression, ${ }^{6}$ and small trials show that third-wave cognitive therapy versus no intervention or treatment as usual is effective for acutely depressed patients. ${ }^{78}$ One trial has shown comparable effects between cognitive therapy and third-wave cognitive therapy in non-melancholic depression, but the trial only included 45 participants. ${ }^{9}$

\section{Mentalisation-based treatment}

Mentalising entails attending to mental states-holding 'mind in mind'. ${ }^{10}$ It is the process by which an individual explicitly and implicitly interpret the action of himself or herself and others on the basis on intentional mental states such as wishes, needs, goals and reason. ${ }^{10}$

Mentalisation-based treatment is rooted in attachment theory and developmental psychopathology and it includes essentials from psychodynamic psychotherapy in a concurrent individual and group format. ${ }^{10}$ Prior to this trial we carried out a systematic review of randomised clinical trials examining the effects of psychodynamic therapy for major depressive disorder. ${ }^{11} \mathrm{We}$ found that psychodynamic therapy versus no intervention seems to have a small statistically significant effect on depressive symptoms (mean difference about three Hamilton Rating Scale for Depression (HDRS) points). ${ }^{11}$ However, we identified a limited number of trials, the trials were small and all the trials had a high risk of bias so our results might be questioned.

Mentalisation-based therapy was originally developed to treat borderline personality disorder but is now also used to treat various other psychiatric disorders such as depression, eating disorders, substance abuse and personality disorders other than borderline. ${ }^{10}{ }^{12}$ Mentalisation-based treatment is based on the concept of mentalisation as described by Fonagy and Bateman, ${ }^{13}$ 14 and is different from the more strictly defined mentalisation-based therapy as manualised by Karterud and Bateman. ${ }^{13-16}$ In comparison with mentalisationbased therapy, mentalisation-based treatment used in this trial has a more open therapeutic stance-letting the patient decide the theme in an associative way. The therapist is less active in directing the theme in the dialogue and uses interpretations. Mentalising deficits can be assumed to underlie depressive symptoms, ${ }^{17}{ }^{18}$ and many depressed patients have a comorbid personality disorder. ${ }^{19}$ We did not identify any trial assessing the effects of mentalisation-based treatment or therapy versus no intervention for major depressive disorder. ${ }^{11}$

\section{Third-wave cognitive therapy versus mentalisation-based treatment}

No randomised clinical trials or systematic reviews seem to have examined the effects of third-wave cognitive therapy versus mentalisation-based treatment or therapy for major depression. ${ }^{20}$

\section{METHODS}

In the following, we briefly describe the methodology of this trial. For details please consult our registered (clinicaltrials.gov: NCT01070134) and published protocol. ${ }^{21}$

\section{Objective}

Our objective was to compare the effect of third-wave cognitive therapy versus mentalisation-based therapy in a small sample of participants with major depressive disorder.

\section{Inclusion of participants}

The trial was conducted at a public psychiatric outpatient clinic only treating patients on sick leave due to a psychiatric disorder. Patients were referred from general practitioners, psychiatrists in private practice and medical and psychiatric departments. No special announcement of the trial was made to the referrers. All patients referred to the psychiatric clinic had a full psychiatric examination by a physician who made the preliminary psychiatric diagnoses (Diagnostic and Statistical Manual-IV-TR, DSM-IV-TR). ${ }^{22}$ Eligible patients were then interviewed by the principal investigator (JCJ) who used the depression part of the structured clinical interview for DSM-IV axis I disorders (SCID I) interview ${ }^{23}$ to assess whether the patient fulfilled the criteria for a major depressive disorder (DSM-IV-TR). ${ }^{22}$ Before randomisation baseline assessments were carried out for all outcome measures and all eligible patients were assessed with the structured clinical Interview for DSM-IV axis II disorders (SCID II).$^{24}$ We chose to perform the SCID II assessments because we wanted to compare personality disorders at baseline in the two intervention groups and to exclude patients with schizotypal personality disorder.

The participant had to meet all of the inclusion criteria and none of the exclusion criteria.

Inclusion criteria

1. Age from 18 years to 65 years.

2. Major depressive disorder, whether fist episode or recurrent (DSM-IV-TR). ${ }^{23}$

3. Beck's Depression Inventory (BDI II) score $>13$ points. ${ }^{25}$

4. Written informed consent.

Exclusion criteria

1. Current psychosis, schizophrenia or schizotypal personality disorder (DSM-IV-TR). ${ }^{22}$

2. A significant alcohol or substance abuse (assessed during the preliminary consultations).

3. Initiated or changed medical antidepressive treatment less than 6 weeks before randomisation.

4. Pregnancy.

5. No written informed consent. 


\section{Randomisation}

Eligible patients with major depressive disorder were randomised 1:1 to third-wave cognitive therapy versus mentalisation-based treatment. The Copenhagen Trial Unit performed the randomisation centrally, using a computer generated block randomisation sequence that was unknown to the investigators. Participant inclusion began in February 2010 and the last patient was randomised in July 2011. Owing to an unequal allocation of the trial participants to one of the two groups in the beginning of the trial (there were only a few participants in one of the groups), the block size was reduced from 12 to 4 and a stratification variable (HDRS score $\geq 22$ points) was removed. The block sizes were at all times unknown to the trial investigators, and the Copenhagen Trial Unit performed these changes without informing the investigators of the changes. Otherwise, the methodology was not changed after trial began.

\section{Interventions}

Each participant received treatment for 18 weeks. The two intervention groups were 'slow-open' (new patients entered the group continually) with a maximum of seven patients per group.

The time of each of the elements in the comprehensive treatment package (see below) was planned to be similar in the compared intervention groups.

\section{Shared elements for both intervention groups}

All participants were, as part of the outpatient clinic's usual care, offered a communal breakfast twice a week and participated in group psychoeducation for $1 \mathrm{~h}$ a week. During the course of treatment, all participants with children were offered participation in a parent support group (four weekly 1 -h sessions). A psychiatric consultant (KAL), who was not otherwise involved in the interventions, assessed each participant and prescribed psychopharmacological treatment when needed. The psychiatric consultant prescribed medication according to the official recommendations. ${ }^{26}$ After the first consultation, medical consultations were offered by demand of the participant or the therapists.

\section{Third-wave cognitive therapy}

The third-wave cognitive therapy consisted of one weekly third-wave cognitive individual psychotherapy session (45 min) and one weekly mindfulness-skills training group $(1.5 \mathrm{~h})$. Altogether the third-wave cognitive therapy consisted of 18 individual psychotherapy sessions (45 min) and 18 group sessions $(1.5 \mathrm{~h})$, a maximal total of $40.5 \mathrm{~h}$.

The weekly individual psychotherapy session included:

- Introduction of the cognitive model and mindfulness.

- Exploration of thoughts, feelings, behaviour and physical sensations.

- Work on acceptance of difficult feelings and difficult life circumstances.
- Work on assumptions challenged by behavioural experiments.

- Self-esteem training.

- Tools to prevent relapse.

The weekly mindfulness-skills training group included:

Education in the practical use of six basic mindfulness skills: focusing, acceptance, labelling feelings, body awareness, self-esteem skills and mindful communication. The group participants were encouraged to practice the six mindfulness skills between sessions. The participants went through the complete skills training group's programme three times during the course of the 18 weeks of treatment.

The manual for the third-wave cognitive therapy was developed specifically for the trial and had not been used before in a trial setting. Details about the third-wave $\operatorname{cog}$ nitive therapy programme is available elsewhere (http:// ctu.dk/publications/supplementary-material.aspx). ${ }^{27}$

\section{Mentalisation-based treatment}

The mentalisation-based treatment consisted of a weekly mentalisation-based individual psychotherapy session (45 min) and a weekly mentalisation-based group therapy session $(1.5 \mathrm{~h})$. Altogether the mentalisationbased treatment consisted of 18 individual psychotherapy sessions $(45 \mathrm{~min})$ and 18 group sessions $(1.5 \mathrm{~h})$, a maximal total of $40.5 \mathrm{~h}$.

Mentalisation-based treatment imposes explicit attention to mentalising in the therapeutic process. This is established by a therapeutic stance where the therapist aims at demonstrating a 'mentalising attitude', that is, validating, 'not-knowing' and curiously questioning the patient about feelings and thoughts. ${ }^{10} 1628$ The therapist tries to identify and intervene when the patient is not mentalising and assists the patient in regulating the level of the emotions so the patient is able to mentalise and to get different perspectives on life events, conflicts etc. ${ }^{10} 1628$

At the time this project was planned there was no manual available for the mentalisation-based treatment. Therefore, we developed our own treatment manual based on mentalisation principles. ${ }^{29}$ Further details about the mentalisationbased treatment is available elsewhere (http://ctu.dk/ publications/supplementary-material.aspx). ${ }^{29}$

\section{Therapists and adherence to the intervention manuals}

Each intervention group had two therapists. The two third-wave cognitive therapists (one of these therapists was the principal investigator) and the two mentalisation-based therapists had comparable psychotherapeutic education and experience.

All individual sessions were recorded on an audio recorder and all group sessions were recorded on video. An experienced external psychologist not otherwise involved in the trial assessed the degree of adherence to the manuals $0-5$ (0: no adherence; 1 : adherence about $20 \%$ of the time; 2: adherence about $40 \%$ of the time; 3 : adherence about $60 \%$ of the time; 4 : adherence about 
$80 \%$ of the time; 5 : adherence about $100 \%$ of the time). The psychologist randomly selected $4 \times 5$ sessions using a computer program. The results showed high adherence to the treatment manuals for both interventions. The means of the ratings were: 4.6 in five sessions of individual third-wave cognitive therapy; 4.2 in five sessions of third-wave cognitive group therapy; 4.2 in five sessions of individual mentalisation-based treatment; and 3.8 in five sessions of mentalisation-based group treatment.

\section{Outcomes}

Primary outcome

- Score on the HDRS ${ }^{30}$ after end of treatment at week 18.

\section{Secondary outcomes}

- The proportion of participants in remission after cessation of treatment at week 18 . We defined remission as HDRS below $8 .^{31}$

- Global Severity Index score (GSI-score) ${ }^{32}$ on the Symptom Checklist 90 Revised (SCL-90-R) ${ }^{32}$ after cessation of treatment at week 18 .

- Score on the WHO-Five Well-being Index 1999 (WHO 5) ${ }^{33}$ after cessation of treatment at week 18.

- Score on the BDI $\mathrm{II}^{25}$ after cessation of treatment at week 18.

\section{Reliability of the HDRS interviews}

Two experienced psychologists performed the Hamilton interviews during the trial period. Prior to the trial, the principal investigator and one of the psychologists both Hamilton interviewed eight patients at the same time point. The mean difference between these two HDRS ratings performed on the same patient at the same time point was -0.13 points (SD 1.25) (intraclass correlation coefficient 0.98; Spearman correlation 0.92). During the trial both psychologists Hamilton interviewed 21 patients at the same time point. The mean difference between these two HDRS ratings performed on the same patient at the same time point was 0.29 points (SD 2.21; intraclass correlation coefficient 0.96; Spearman correlation 0.94). All these 29 interviews were performed with both HDRS-raters present simultaneously. One rater interviewed and rated the interviewee and the other rater only rated the interviewee. The interviewers were not allowed to discuss the results before each interviewer had registered the HDRS result.

\section{Data-management}

All data were handled by research assistants not otherwise involved in the trial and was stored in the principal investigator's office and later at the Copenhagen Trial Unit. Privacy of trial participants was protected in accordance with the Act on Processing of Personal Data and the Health Act. The project was notified to the Danish Data Protection Agency (no. 2008-58-0020).

\section{Blinding}

The Hamilton interviewers were blinded to treatment allocation and were instructed by the principal investigator to avoid questions beside the Hamilton interview. All interviewees were prior to each interview instructed by the principal investigator not to mention which treatment they were allocated to. It was not possible to blind neither the therapists nor the participants to treatment allocation.

The chief consultant performing the medical consultations was, due to practical circumstances, not blinded to treatment allocation.

A statistician at The Copenhagen Trial Unit performed the statistical analyses blinded with the two intervention groups coded as 'A' and 'B'.

\section{A priori sample size estimate}

With a 'minimal relevant mean difference' (MIREDIF) between the two interventions of 5 HDRS points, an $\alpha$ of 0.05 (type I error), a power of 0.90 (type II error of $10 \%)$ and a SD of 7 HDRS points, the sample size calculation showed that a total of 84 participants would be necessary. We estimated that we would need an inclusion period of about 2 years to recruit 84 participants.

\section{Statistical analyses}

The primary analyses were intention-to-treat analyses. Significance tests were two-sided at a significance level of 0.05 .

Continuous outcomes were compared between the two intervention groups using the univariate general linear model with (ANCOVA) and without HDRS baseline value adjustment (ANOVA). The binary outcome was compared between the groups using Fisher's exact test. Logistic regression could not be used since none of the participants in the mentalisation-based group obtained remission implying an infinite OR.

As the trial was stopped before the sample size was reached, we post hoc decided to conduct sequential analysis to assess the results of significance testing taking sparse data and repetitive testing into consideration. ${ }^{34}$ We used the trial sequential analysis programme for that purpose. $^{35-38}$

\section{RESULTS}

\section{Participants}

Only 44 out of the 84 planned participants were included in the trial. Twenty-two participants were randomised to third-wave cognitive therapy versus 22 participants to mentalisation-based treatment. Figure 1 details the participant flow through the phases of the trial.

\section{Baseline characteristics of the participants}

The baseline characteristics regarding age, sex, number of children, score on the HDRS, baseline diagnosis of personality disorder and psychopharmacological treatment were overall assessed as being comparable between 


\section{CONSORT 2010 Flow Diagram}

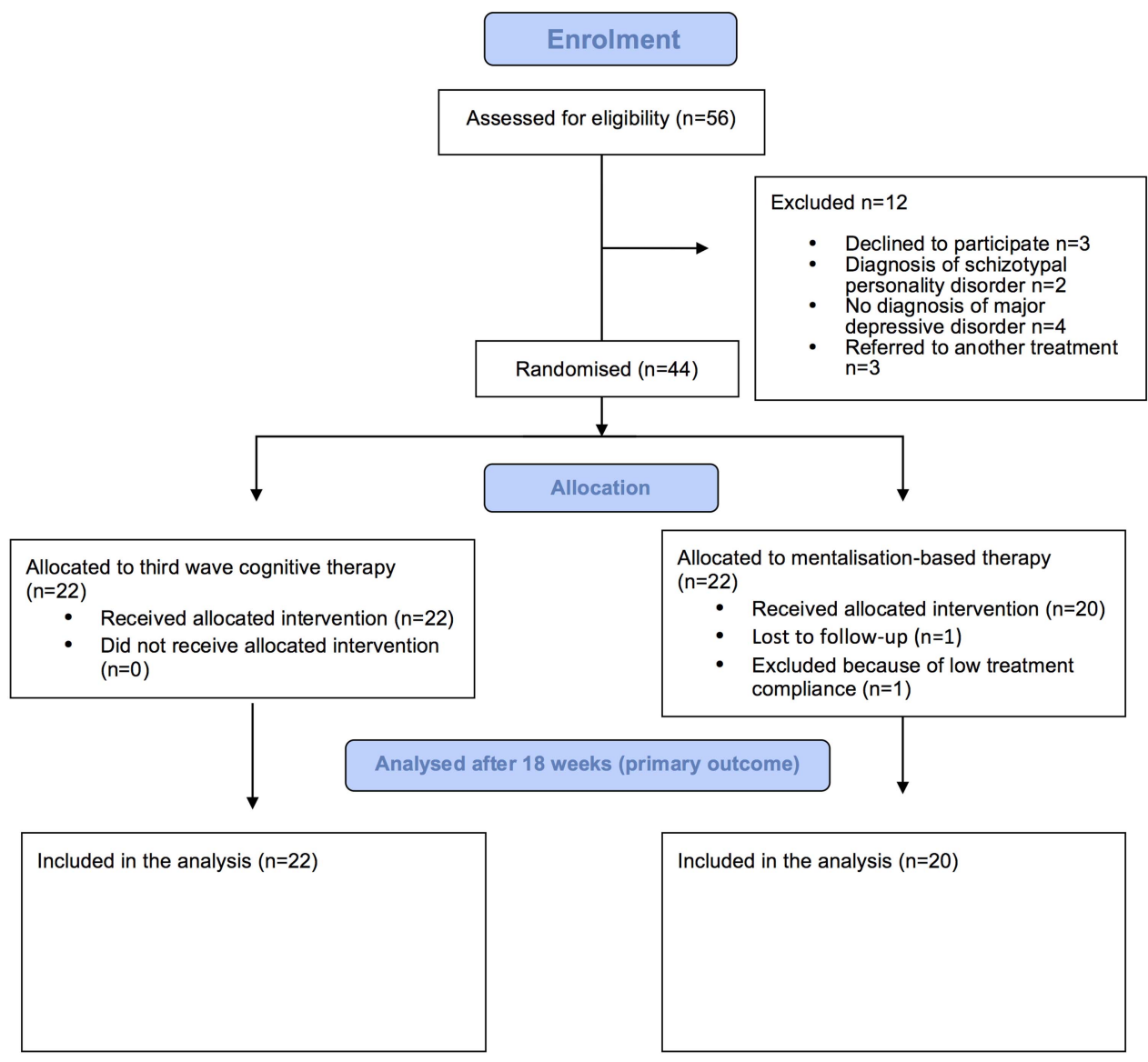

Figure 1 CONSORT flow chart.

the two intervention groups. The baseline participant characteristics are described in detail in table 1 and the psychopharmacological treatment in online supplementary material 1.

\section{Treatment compliance}

None of the 22 participants randomised to third-wave cognitive therapy were lost to follow-up or excluded due to the fact that they participated in less than $70 \%$ of the sessions. One participant out of the 22 randomised to mentalisation-based treatment was lost to follow-up and one was excluded, as she did not attend the required $70 \%$ of the sessions (figure 1). The excluded participant was not assessed on any of the outcomes at end of treatment.

\section{INTERVENTION EFFECTS}

\section{Primary outcome}

Mean score on the HDRS after end of interventions

Participants randomised to third-wave therapy compared with participants randomised to mentalisation-based treatment did not differ significantly regarding the 18-week HDRS scores in the unadjusted analysis (mean $12.9,95 \%$ CI 9.81 to 15.9 vs mean $17.0,95 \%$ CI 14.0 to $20.0 ; \mathrm{p}=0.051)$. The mean difference between the two groups was -4.14 HDRS points (95\% CI -8.30 to 0.03 ) corresponding to a Cohen's D of -0.62 . The difference was, however, significant in the analysis adjusted for baseline HDRS score ( $\mathrm{p}=0.039$; table 2$)$.

Sequential analysis demonstrated that the observed significant findings ought to be interpreted conservatively as random errors due to sparse data cannot be excluded (figure 2).

We did not impute missing values because only 2 out of $44(4.5 \%)$ participants had missing values.

Histograms on the data from both intervention groups showed that the data seem to be normally distributed. Using the non-parametric test the $\mathrm{p}$ value was 0.064 .

There was no significant interaction between the indicator of a diagnosis of a personality disorder and the intervention effects. This was also the case when the indicator was redefined as a binary quantity defined as any kind of personality disorder (yes/no) or as a binary quantity defined as personality disorder=borderline personality disorder (yes/no).

\section{Secondary outcomes}

Participants in remission after cessation of treatment

In the third-wave cognitive therapy group $22.7 \% \quad(n=5)$ were in remission after cessation of treatment (defined 


\begin{tabular}{|c|c|c|}
\hline & $\begin{array}{l}\text { Participants randomised to third-wave } \\
\text { cognitive therapy }(n=22)\end{array}$ & $\begin{array}{l}\text { Participants randomised to } \\
\text { mentalisation-based therapy }(n=22)\end{array}$ \\
\hline \multicolumn{3}{|l|}{ Age } \\
\hline Mean (SD) & $38.5(8.9)$ & $40.3(6.8)$ \\
\hline \multicolumn{3}{|l|}{ Sex } \\
\hline Female, n (\%) & $18(82)$ & $20(91)$ \\
\hline \multicolumn{3}{|l|}{ Number of children } \\
\hline Mean (SD) & $1.4(1.2)$ & $1.7(1.1)$ \\
\hline \multicolumn{3}{|l|}{ Marital status, n (\%) } \\
\hline Single & $3(14)$ & 7 (32) \\
\hline In a relationship & $6(27)$ & $5(23)$ \\
\hline Married & $12(55)$ & $8(36)$ \\
\hline Separated/divorced & $1(5)$ & $2(9)$ \\
\hline \multicolumn{3}{|l|}{ Level of education, n (\%) } \\
\hline Only high school diploma & $7(32)$ & $3(14)$ \\
\hline Medium long education & $14(64)$ & $19(86)$ \\
\hline Long education & $1(5)$ & $0(0)$ \\
\hline \multicolumn{3}{|l|}{ Baseline HDRS scores } \\
\hline Mean (SD) & $22.1(5.9)$ & $22.5(4.9)$ \\
\hline Median & 22.5 & 23.6 \\
\hline Range & $7-30$ & $11-29$ \\
\hline \multicolumn{3}{|l|}{ Baseline GSI scores (SCL 90-R) } \\
\hline Mean (SD) & $1.80(0.59)$ & $1.84(0.41)$ \\
\hline Median & 1.72 & 1.74 \\
\hline Range & $0.68-2.79$ & $0.99-2.54$ \\
\hline \multicolumn{3}{|l|}{ Personality disorders, $n(\%)$} \\
\hline No personality disorder & $5(23)$ & $6(27)$ \\
\hline One personality disorder & $11(50)$ & $12(55)$ \\
\hline Two personality disorders & $4(18)$ & $3(14)$ \\
\hline Three or more personality disorders & $2(9)$ & $1(5)$ \\
\hline \multicolumn{3}{|l|}{ Personality disorders diagnoses, n (\%) } \\
\hline Paranoid & $1(5)$ & $0(0)$ \\
\hline Borderline & $4(18)$ & $1(5)$ \\
\hline Avoidant & $7(32)$ & $5(23)$ \\
\hline Obsessive-compulsive & $4(18)$ & $3(14)$ \\
\hline Dependant & $1(5)$ & $0(0)$ \\
\hline Depressive & 7 (32) & $8(36)$ \\
\hline Personality disorder NOS & $1(5)$ & $4(18)$ \\
\hline
\end{tabular}

as having $\mathrm{HDRS}<8$ points) versus $0 \%$ in the mentalisation-based treatment group. This difference was significant $(\mathrm{p}=0.049$; table 2$)$.

BDI II, ${ }^{25}$ SCL-90-R ${ }^{32}$ and WHO $5^{33}$ after end of interventions No significant difference was found on BDI II, SCL-90-R (GSI-scores), or WHO 5 between the two intervention groups after cessation of treatment (table 2). Sequential analysis demonstrated that the observed insignificant findings ought to be interpreted conservatively as random errors due to sparse data cannot be excluded (see figure 3 regarding BDI II).

\section{Other outcomes}

Admissions, suicide attempts and suicides

One of the participants randomised to third-wave cognitive therapy and two of the participants randomised to mentalisation-based treatment were for a short period (some days) admitted to a psychiatric hospital during the intervention period.

We recorded no suicide attempts or suicides during the intervention period in any of the 44 participants.

\section{DISCUSSION}

Our preliminary results indicate that third-wave cognitive therapy compared with mentalisation-based treatment may be a more effective intervention for lowering depressive symptoms measured on the HDRS and may increase the probability of remission (HDRS $<8$ points). Furthermore, our trial demonstrated the feasibility of conducting the trial with low risks of bias. However, when only 44 out of the planned 84 participants $(52 \%)$ of the projected sample size is obtained in a trial, it is necessary to interpret the results cautiously. Had this 
Table 2 Effects of third-wave cognitive therapy versus mentalisation-based treatment

\begin{tabular}{|c|c|c|c|c|c|c|}
\hline \multirow[b]{2}{*}{ Outcome measure } & \multicolumn{2}{|c|}{$\begin{array}{l}\text { Group randomised to } \\
\text { third-wave cognitive } \\
\text { therapy }(\mathrm{N}=22)\end{array}$} & \multicolumn{2}{|c|}{$\begin{array}{l}\text { Group randomised to } \\
\text { mentalisation-based } \\
\text { treatment }(\mathrm{N}=22)\end{array}$} & \multirow{2}{*}{$\begin{array}{l}\text { p Value of } \\
\text { unadjusted } \\
\text { analysis at end } \\
\text { of treatment }\end{array}$} & \multirow{2}{*}{$\begin{array}{l}p \text { Value of } \\
\text { adjusted } \\
\text { analysis }{ }^{\star} \text { at end } \\
\text { of treatment }\end{array}$} \\
\hline & Baseline & $\begin{array}{l}\text { End of } \\
\text { treatment }\end{array}$ & Baseline & $\begin{array}{l}\text { End of } \\
\text { treatment }\end{array}$ & & \\
\hline \multicolumn{7}{|l|}{ HDRS } \\
\hline $\mathrm{N}$ & 22 & 22 & 21 & 20 & 0.051 & 0.039 \\
\hline Mean & 22.1 & 12.9 & 22.5 & 17.0 & & \\
\hline $95 \% \mathrm{Cl}$ & 19.5 to 24.8 & 9.81 to 15.9 & 20.3 to 24.8 & 14.0 to 20.0 & & \\
\hline $\begin{array}{l}\text { Remission } \\
\text { (HDRS<8) N/total } \\
\text { BDI II }\end{array}$ & $0 / 22$ & $5 / 22$ & $0 / 21$ & $0 / 20$ & 0.049 & $\begin{array}{l}\text { Not possible to } \\
\text { calculate }\end{array}$ \\
\hline $\mathrm{N}$ & 21 & 21 & 22 & 17 & 0.46 & 0.46 \\
\hline Mean & 36.8 & 17.6 & 36.3 & 20.5 & & \\
\hline $95 \% \mathrm{Cl}$ & 32.5 to 41.1 & 12.2 to 23.0 & 32.1 to 40.6 & 14.5 to 26.4 & & \\
\hline \multicolumn{7}{|c|}{ SCL 90-R (GSI score) } \\
\hline $\mathrm{N}$ & 22 & 22 & 22 & 20 & 0.52 & 0.66 \\
\hline Mean & 1.80 & 0.88 & 1.84 & 1.00 & & \\
\hline $95 \% \mathrm{Cl}$ & 1.54 to 2.05 & 0.62 to 1.15 & 1.66 to 2.02 & 0.74 to 1.25 & & \\
\hline \multicolumn{7}{|l|}{ WHO 5} \\
\hline $\mathrm{N}$ & 22 & 22 & 21 & 20 & 0.54 & 0.46 \\
\hline Mean & 3.55 & 10.5 & 4.33 & 9.45 & & \\
\hline $95 \% \mathrm{Cl}$ & 1.84 to 5.25 & 7.66 to 13.4 & 3.13 to 5.53 & 7.18 to 11.7 & & \\
\hline
\end{tabular}

been an interim analysis, any independent safety and data monitoring committee would have recommended continued randomisation and completion of the trial (figures 2 and 3). ${ }^{34}$ Furthermore, the two interventions do not seem to have significant differential effects on BDI (subjective depressive symptoms), SCL 90-R (psychological distress) and WHO 5 (well-being).

Compared with the baseline scores, both intervention groups improved during the trial period on all continuous outcomes. We did not include a control group receiving no intervention in this head-to-head trial so it is unclear whether it was trial intervention effects, regression towards the mean or the natural progression of the disorder in this sample which was responsible for these changes. ${ }^{39}$ More randomised clinical trials are needed to assess the effects of third-wave cognitive therapy and mentalisation-based treatment for major depressive disorder.

\section{Strengths}

First of all, the trial was conducted with an overall high level of methodological quality and we assessed the validity of the trial results according to the procedure proposed by Jakobsen et $a l^{4}$, including adjusting the thresholds for significance according to the number of randomised participants and the planned sample size. We also proved the feasibility of our trial design, which can be used for larger trials provided that funding can be raised. Our trial has a number of additional strengths: (1) The trial protocol was registered before randomisation began at ClinicalTrials.gov. In this protocol the outcome hierarchy and plans for analyses were presented. Our trial was altogether conducted according to good clinical research practice, with low risk of bias (adequate allocation sequence generation, adequate allocation concealment, adequate blinding, no risk of selective outcome reporting, low risk of incomplete outcome data bias, no risk of 'for profit' bias) and a high degree of external validity. ${ }^{40-44}$ (2) Both of the trial interventions were conducted using manuals (available at http://ctu.dk/publications/supplementary-material. aspx) and adherence to the manuals was assessed as relatively high by an independent Danish psychologist trained in mentalisation-based therapy and third-wave cognitive therapy. The manualisation of the trial interventions makes it possible, to some extent, to implement the two trial interventions in clinical practice and to replicate or refute our results in future trials, but both treatment manuals are currently only available in Danish, which limits the possibility for non-Danish speakers to assess the quality of the treatment manuals. We are in the process of translating the third-wave cognitive manual, which will be published at a later time point. The mentalisation-based treatment is described thoroughly elsewhere. ${ }^{13-16}$ Nevertheless, it is a clear limitation that the manuals are not currently available in English. Both the cognitive therapists and the mentalisation therapists were involved in developing the treatment manuals for the respective psychotherapeutic treatments, which might make the therapist enthusiasm and 
Figure 2 Post hoc sequential analysis of the results on the Hamilton depression rating scale (HDRS) after 18 weeks. Forty-two participants of the 44 participants were assessed with HDRS after end of treatment. The required information size of 83 participants is calculated based on minimal relevant mean difference of 5 HDRS points, a type I error of $5 \%$, a $\beta$ of $10 \%$ (power of $90 \%$ ) and a variance of $49 .{ }^{35-38}$ These assumptions are similar to the assumptions used in prospectively planned sample size calculation of 84 participants. The cumulated Z-curve (blue curve) do not cross the sequential monitoring boundaries (red inner sloping lines) implying that there is a risk of random error due to sparse data in the estimate of a beneficial effect of third-wave mentalisation-based therapy. cognitive therapy compared with

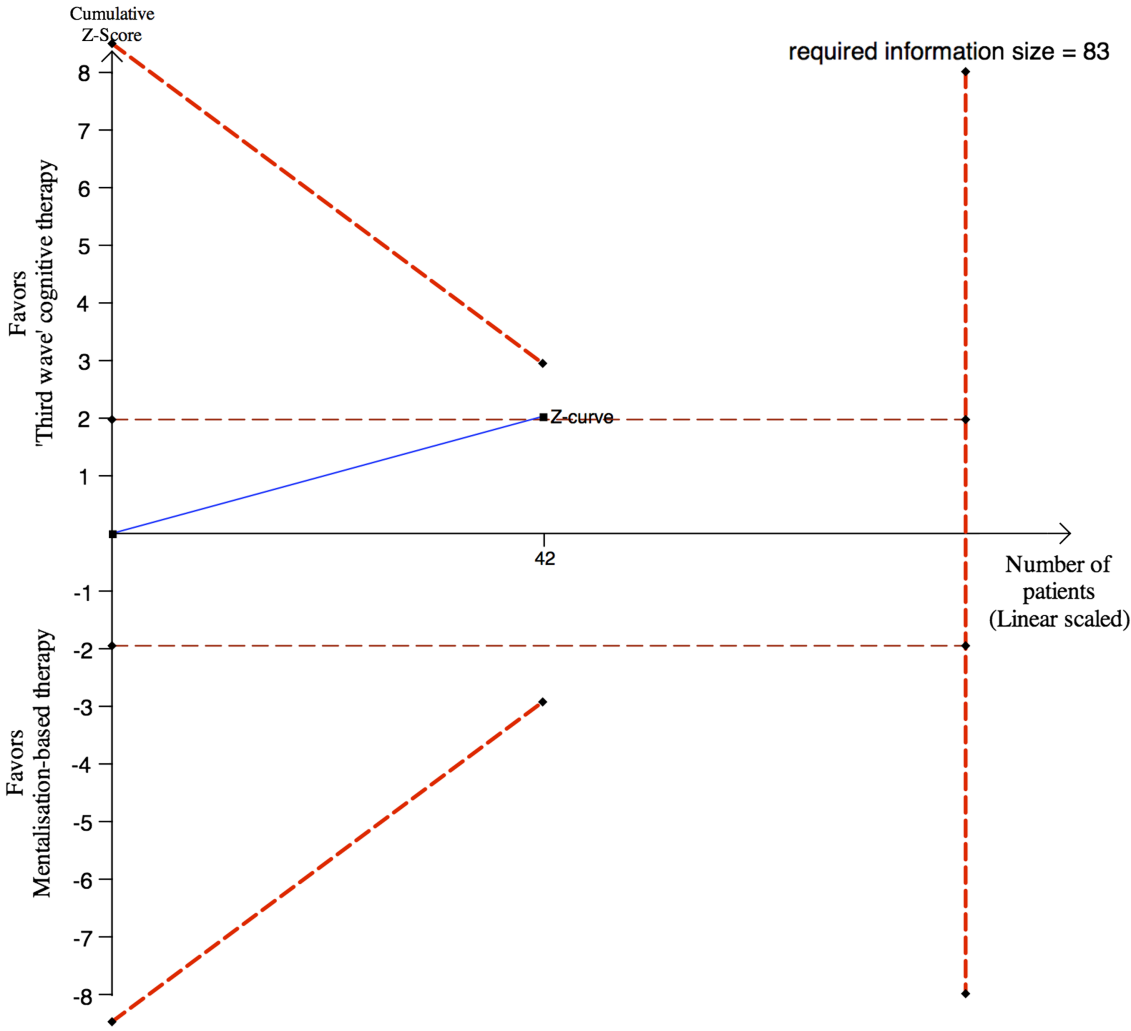

thoroughness similar in the two intervention groups. (3) We have used the most commonly used outcomes in trials assessing the effects of psychotherapeutic interventions for depression (ie, HDRS and BDI). ${ }^{11} 304546$ This makes it possible to relate our results to results from other trials examining the effects of interventions for depression. Moreover, using HDRS as outcome makes it possible to perform blinded objective outcome assessment, which is a further strength of our trial. (4) The baseline characteristics of the trial participants as well as
Figure 3 Post hoc sequential analysis of the results on Beck's depression inventory II (BDI II) after 18 weeks. Thirty-eight of the 44 participants were assessed with BDI II after end of treatment. The required information size of 222 participants is calculated based on minimal relevant mean difference of $5 \mathrm{BDI}$ II points, a type I error of $5 \%$, a $\beta$ of $10 \%$ (power of $90 \%$ ) and a SD of 11.5 BDI II points. ${ }^{35-38}$ The cumulated Z-curve (blue curve) do not cross the sequential monitoring boundaries (red inner sloping lines) implying that there is a risk of random error due to sparse data in the estimate of no beneficial effect of third-wave cognitive therapy compared with mentalisation-based.

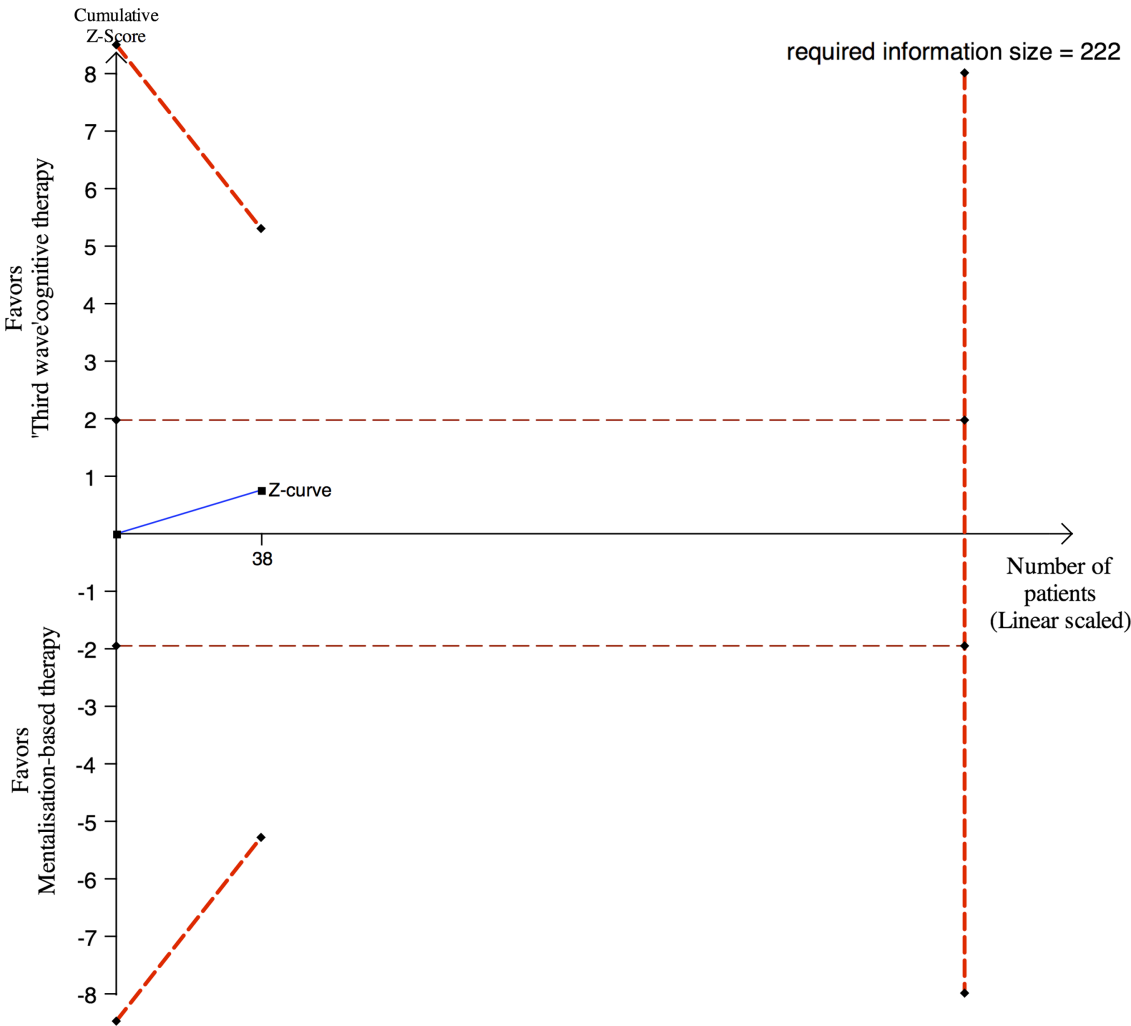


the psychopharmacological medication in the two groups were comparable which indicates that the randomisation succeeded in allocating comparable participants to the two intervention groups. (5) Only 2 out of the total of 44 participants were not assessed after end of treatment, which decreases the risk of biased results. ${ }^{47}$ (6) All outcomes suggested that the participants randomised to third-wave cognitive therapy had improved more than the participants randomised to mentalisationbased treatment. This supports the validity of our results, even though most of these differences were non-significant.

\section{Limitations}

Our trial has a number of limitations. This small-scale trial was in essence failed because we only included 44 out of the planned 84 participants. The trial inclusion lasted for about 2 years as planned but we had problems with recruiting participants. Basically, not enough eligible depressed patients were referred to the clinic within the planned trial period. The great advantage of the randomised clinical trial in general is that all known and unknown participant characteristics will be similar at baseline in compared intervention groups. ${ }^{39}$ However, even though our baseline characteristics indicate similarity between the two groups on assessed baseline characteristics, it is unlikely that all baseline characteristics will be similar when only 44 participants are randomised. The low number of randomised participants in this small-scale trial increases the risks of wrong results due to type I errors, and type II errors ${ }^{48} 49$ and our adequate trial methodology cannot necessarily compensate for these increased risks. Moreover, our results do not show anything about long-term effects of the two interventions.

The chief consultant prescribing the psychopharmacological treatment was not blinded to intervention allocation. Although we assessed the psychopharmacological treatment to be comparable in the two randomised groups at cessation of the trial interventions (see online supplementary material 1), the lack of blinding might have influenced the psychopharmacological treatment. The chief consultant is a mentalisation-based therapist and was involved in developing the mentalisation-based treatment manual. The first author and primary investigator conducted the third-wave cognitive therapy and wrote the manual for the third-wave cognitive therapy programme, which may also increase the risks of bias.

We did not perform power calculations for the secondary outcomes before randomisation began, which is a further limitation. If an analysis of a secondary outcome has a power of less than $80 \%$, then either the secondary outcome should be classified as an exploratory outcome or the $\mathrm{p}$ value and the CI thresholds for significance should be adjusted, just as the thresholds are adjusted if a sample size has not been reached. ${ }^{34}$

Owing to an unequal allocation of the trial participants to one of the two groups in the beginning of the trial, the block size was reduced from 12 to 4 (see 'Randomisation' section). The block sizes were at all times unknown to the trial investigators, and the Copenhagen Trial Unit performed these changes without informing the investigators. However, a block size of four is small making it possible to foresee which group a given eligible participant will be allocated to before randomisation. This might question whether the allocation concealment was effective.

The trial was conducted at an outpatient psychiatric clinic with special interest for treatment of personality disorders and depressive patients were not routinely refereed to the clinic before the trial began randomisation. Our results showed that a high proportion of the trial participants had comorbid personality disorder and depression. This might explain why the baseline HDRS scores indicated that the trial participants were only moderately depressed although all of the trial participants were on sick leave due to psychological problems. Some of the trial participants might suffer primarily from psychological problem other than depressive symptoms, that is, personality-related problems. We did not assess number of prior depressive episodes in the included participants, which makes it unclear whether our trial results demonstrate intervention effects in participants with a first time depression or recurrent depression. Our results can only be related to patients comparable to our trial participants, that is, patients diagnosed with major depressive disorder on sick leave due to psychiatric problems.

Highly specialised mentalisation-based treatment was the primary psychotherapeutic method used at the outpatient clinic prior to the trial, the cointerventions (communal breakfast and psychoeducation) were also a part of the treatment programme prior to the trial, and experienced and specialised third-wave cognitive therapists were members of the staff at the psychiatric clinic where the trial was conducted. Furthermore, all patients refereed to the psychiatric clinic were on sick leave due to psychiatric problems, and even though the evidence behind the specialised treatments is lacking we considered that some form of specialised treatment was needed for all patients at the psychiatric clinic. We did, therefore, not consider it ethically justifiable to use a control group receiving no intervention, placebo or only the cointerventions. All these considerations and practical circumstances led to the choice of the psychotherapeutic interventions and the design of this head-to-head trial comparing third-wave cognitive therapy and cointerventions versus mentalisation-based therapy and cointerventions. The cointerventions where delivered similarly to both treatment groups and the possible effects of cointerventions will therefore even out between the compared intervention groups unless there are significant interactions. Nevertheless, it is a clear limitation that our interventions are not and have not been compared versus no intervention or a more simple and basic form of psychotherapy plus cointerventions. ${ }^{39}$ If a trial 
comparing the effects of two active interventions shows no difference in effect it is not clear whether the two interventions are equally effective or equally ineffective -and if an experimental intervention seem superior compared with a control intervention then the effect size of the experimental intervention will be unclear because any beneficial or harmful effects of the control intervention might influence the trial results. ${ }^{39}$ All interventions should be assessed versus no intervention before being introduced into clinical practice. ${ }^{39}$ Furthermore, the combination of specialised psychotherapy and cointerventions constitute a relatively comprehensive treatment, which might not always be accessible to psychiatric patients in clinical practice-this might limit the generalisability of our results.

\section{Mentalisation-based treatment}

We did not find any relevant treatment manual we could use for the mentalisation-based treatment, and we therefore created our own manual (http://ctu.dk/ publications/supplementary-material.aspx).$^{29}$ The therapists in the mentalisation-based treatment group were educated and experienced in psychodynamic therapy and group therapy and had underwent basic training and education in mentalisation-based treatment according to Bateman and Karterud. ${ }^{13-16}$ Mentalisation-based treatment was originally designed to treat borderline personality. ${ }^{10}{ }^{12}$ Few participants were diagnosed with borderline personality disorder (table 1), and it can be argued that mentalisation-based treatment was not a relevant intervention for the depressed participants of this trial. However, mentalisation-based treatment is now used to treat a number of different disorders other than borderline personality disorder, including depression. ${ }^{10}{ }^{12}$ Furthermore, a study has shown that female inpatients with depression showed a significantly lower capacity for mentalisation compared with healthy controls-and deficits in mentalising capacity were related to illness duration, number of admissions and cognitive impairment. ${ }^{17}$ The authors conclude that the investigation of mentalisation may be of particular importance for the development of targeted psychotherapeutic interventions for depression. ${ }^{17}$

\section{Comorbid personality disorders}

A large proportion of the included participants were diagnosed with cluster $\mathrm{C}$ personality disorders (anxious or fearful personality disorders). ${ }^{23}{ }^{24}$ It has been debated if a diagnosis of a personality disorder is accurate when patients are acutely depressed. ${ }^{19}$ Our results indicate that comorbid personality disorder and depression does not lead to a poorer outcome compared to patients with depression alone-but this could be because the diagnoses of the personality disorders in our trial are inaccurate because the depressive symptoms might mimic pathological personality traits. Furthermore, the limited number of included participants significantly reduces the power of this analysis.

\section{BDI compared to HDRS as outcome}

It is a common belief among clinicians that BDI is a more 'reactive' outcome than HDRS, ${ }^{50}$ and it might be surprising to some why we identified a borderline significant effect on the HDRS results but no significant effect on the BDI. However, two systematic reviews with meta-analysis have included trials that simultaneously used HDRS and BDI to assess the effects of the same interventions. $^{50} 51$ The results showed that BDI under such circumstances shows significantly less effect sizes compared to the HDRS. ${ }^{50}{ }^{51}$ A greater percentage of participants would be considered improved if ratings of change were based on the HDRS rather than BDI. ${ }^{50}$ The results from these two reviews ${ }^{50}$ are in agreement with our present results and may explain why we found a borderline significant effect on HDRS and no significant effect on BDI. On the other hand, it is also possible that HDRS compared to BDI overestimates participant improvement. ${ }^{51}$

It was impossible to blind the participants to treatment allocation. To ensure some degree of blinding we chose HDRS over BDI because it was possible to perform objective blinded outcome assessment using the HDRS. BDI is a self-administered questionnaire, which makes blinded objective outcome assessment impossible. We therefore expected the results on HDRS to be a more clinically valid compared to the BDI results-but we cannot exclude that breaking of blinding and biased assessment of the HDRS may have occurred. In accordance with the CONSORT Statement we did not assess degree of unblinding. ${ }^{40}$

\section{Implications}

First of all, if a larger more definitive trial has to be conducted then a more realistic estimate of the recruitment rate will be needed and more centres should be involved. On average, we recruited approximately one participant every third week and we expected to be able to recruit approximately one participant every week. Basically, not enough eligible participants were referred to the clinic during the inclusion period and we had to terminate the trial due to economical and practical constraints-this was the primary reason why we did not randomise more participants. Before the randomisation began, we did not systematically assess how many participants it was possible to recruit. This should also be carried out before a larger trial is conducted so the sample size can be reached. Moreover, we did not take any specific actions promoting the trial outside the clinic. If a future trial is to be conducted it should be considered to promote the trial through advertising or use of other measures to motivate potential referrers to refer more eligible participants. Besides the problems with recruiting enough participants, it was otherwise feasible to conduct a randomised clinical trial with low risk of bias assessing the effects of third-wave cognitive therapy versus mentalisation-based treatment for major depressive disorder. 
The apparent difference in intervention effect found on the HDRS might be caused by random error ("play of chance'), unaccounted bias or a signal of a real effect. ${ }^{49}$ The National Institute for Health and Care Excellence (NICE) have suggested a mean difference between two compared interventions of three HDRS points as a criterion for 'clinical significance'. ${ }^{52}$ Most interventions for depression, both psychopharmacological as well as psychotherapeutic, rarely exceed having a beneficial effect of more than three HDRS points. ${ }^{1}{ }^{11}$ ${ }^{53-55}$ We used an anticipated intervention effect of five HDRS points to estimate the necessary sample size and this anticipated intervention effect was optimistic. Calculating Bayes factor based on the anticipated intervention effect, the observed intervention effect, and the $\mathrm{SE}$ of the observed intervention effect shows a Bayes factor of 0.14 , which is above the recommended threshold for significance of $0.1 .^{34}$ This underlines that our results should be regarded as insignificant and that an anticipated intervention effect lower than five HDRS points ought to be used in sample size calculations in future trials assessing the effects of third-wave cognitive therapy and mentalisation-based therapy. We found a mean difference of more than four HDRS points which, compared to other interventions, is relatively high. These results might be used to calculate a necessary sample size in a larger more definitive trial. However, HDRS might not at all be a clinically relevant outcome and other more clinically relevant outcomes might be more valid to use in future trials. Severity of depression as measured by the total HDRS score has failed to predict suicide attempts, ${ }^{56} 57$ and some publications have questioned the usefulness of the HDRS and concluded that the scale is psychometrically and conceptually flawed. 5758

\section{CONCLUSIONS}

Our preliminary results show that third-wave cognitive therapy compared with mentalisation-based treatment may be a more effective intervention for depressive symptoms measured on the HDRS. The effects of the two interventions did not seem to differ significantly regarding BDI II, SCL 90-R and WHO 5. More randomised clinical trials are needed to assess the effects of third-wave cognitive therapy and mentalisation-based treatment.

\footnotetext{
Author affiliations

${ }^{1}$ Psychiatric Research Unit, Copenhagen University Hospital, Copenhagen, Denmark

${ }^{2}$ Copenhagen Trial Unit, Centre for Clinical Intervention Research, Department 7812, Rigshospitalet, Copenhagen University Hospital, Copenhagen, Denmark ${ }^{3}$ Department of Psychiatry, Psychiatric Clinic, Roskilde, Denmark

${ }^{4}$ Mental Health Services, Psychotherapy Center Stolpegaard, Copenhagen, Denmark

${ }^{5}$ Department of Public Health, University of Copenhagen, Copenhagen, Denmark

${ }^{6}$ Institute of Clinical Medicine, Faculty of Health Sciences, University of Copenhagen, Copenhagen, Denmark
}

Acknowledgements The authors would like to thank all the participants in the trial for patiently cooperating with the assessments. The authors would also like to thank Anita Jensen for helping with data management; Lotte Dragsted and Marianne Lyngby for performing the Hamilton interviews; Gitte Nielsen for assistance in developing the treatment manual for the third-wave cognitive therapy; and Jane Lindschou for expert assistance with the randomisation. Lastly, the authors would like to thank all the coworkers at the psychiatric clinic in Roskilde. Without their patience and cooperation the trial would have been impossible to conduct.

Contributors JCJ and CG wrote the first draft. JCJ, CG, KAL, PS, US and ES planned and designed the trial. JCJ and MK performed the reliability tests. KAL, PS, US and ES contributed with psychiatric expertise. PW conducted the statistical analyses. TL contributed with statistical expertise. All authors contributed academically to the manuscript and have accepted the manuscript for publication.

Funding We have received external funding for the trial from the Health Science Fund, Region Zealand, Denmark (governmental funding). There were no commercial sponsors.

Competing interests The principal investigator was also a therapist in the third-wave cognitive therapy treatment and has developed the treatment manual for the third-wave cognitive therapy. The consultant performing the medical consultations during the trial period was not blinded to the treatment allocation of the participants and developed the mentalisation-based treatment manual in close cooperation with the two mentalisation-based therapists. Other authors have no competing interests.

\section{Patient consent Obtained.}

Ethics approval Before randomisation began approval was obtained by the Regional Ethics Committee of Zealand (no: SJ-43) and the trial was registered at the Danish Data Protection Agency (no: 2008-58-0020).

Provenance and peer review Not commissioned; externally peer reviewed.

Data sharing statement Extra data can be accessed via the Dryad data repository at http://datadryad.org/ with the doi:10.5061/dryad.2d7h5.

Open Access This is an Open Access article distributed in accordance with the Creative Commons Attribution Non Commercial (CC BY-NC 3.0) license, which permits others to distribute, remix, adapt, build upon this work noncommercially, and license their derivative works on different terms, provided the original work is properly cited and the use is non-commercial. See: http:// creativecommons.org/licenses/by-nc/3.0/

\section{REFERENCES}

1. Jakobsen JC, Hansen JL, Storebø OJ, et al. The effects of cognitive therapy versus 'no Intervention' for major depressive disorder. PLoS ONE 2011;6:e28299.

2. Kahl KG, Winter L, Schweiger U, et al. The third wave of cognitive-behavioural psychotherapies: concepts and efficacy. Fortschr Neurol Psychiatr 2011;79:330-9.

3. Hofmann SG, Sawyer AT, Witt AA, et al. The effect of mindfulnessbased therapy on anxiety and depression: a meta-analytic review. $J$ Consult Clin Psychol 2010;78:169-83.

4. Linehan MM, Comtois KA, Murray AM, et al. Two year randomized controlled trial and follow-up of dialectical behavior therapy vs therapy by experts for suicidal behaviours an borderline personality disorder. Arch Gen Psychiatry 2006;63:757-66.

5. Krisanaprakornkit T, Ngamjarus C, Witoonchart C, et al. Meditation therapies for attention-deficit/hyperactivity disorder (ADHD). Cochrane Database Syst Rev 2010(6):CD006507.

6. Coelho HF, Canter PH, Ernst E. Mindfulness-based cognitive therapy: evaluating current evidence and informing future research. $J$ Consult Clin Psychol 2007;75:1000-5.

7. Thompson NJ, Walker ER, Obolensky N, et al. Distance delivery of mindfulness-based cognitive therapy for depression: project UPLIFT. Epilepsy Behav 2010;19:247-54.

8. Barnhofer T, Crane C, Hargus E, et al. Mindfulness-based cognitive therapy as a treatment for chronic depression: a preliminary study. Behav Res Ther 2009;47:366-73.

9. Manicavasgar V, Parker G, Perich T. Mindfulness-based cognitive therapy vs cognitive behaviour therapy as a treatment for non-melancholic depression. J Affect Disord 2011:138-44. 
10. Bateman A, Fonagy P. Mentalization based treatment for borderline personality disorder. World Psychiatry 2010;9:11-15.

11. Jakobsen JC, Hansen JL, Simonsen E, et al. The effect of adding psychodynamic therapy to antidepressants in patients with major depressive disorder. A systematic review with meta-analyses and trial sequential analyses. J Affect Disord 2012;137:4-14.

12. Bateman A, Fonagy P. Randomized controlled trial of outpatient mentalization-based treatment versus structured clinical management for borderline personality disorder. Am J Psychiatry 2009;166:1355-64

13. Bateman A, Fonagy P. Psychotherapy for borderline personality disorder, mentalisation based treatment. Oxford University Press, 2004.

14. Bateman A, Fonagy P. Mentalisation-based treatment for borderline personality disorder - a practical guide. Oxford University Press, 2006.

15. Karterud S. Manual for mentaliseringsbaseret gruppeterapi (MBT-G) (in Norwegen) (manual for mentalisation-based group therapy). Gyldendal Norsk Forlag 2012.

16. Karterud S, Bateman A. Manual for Mentaliseringsbasert psykedukativ gruppeterapi (MBT-I) (Manual for mentalization-based group therapy. Saxo.com (2011) 2011. http://www.saxo.com/dk/ manual-for-mentaliseringsbasert-psykedukativ-gruppeterapi-mbt-i_ sigmund-karterud haeftet 9788205419674

17. Fischer-Kern M, Fonagy P, Kapusta ND, et al. Mentalizing in female inpatients with major depressive disorder. $J$ Nerv Ment Dis 2013;201:202-7.

18. Cusi AM, Nazarov A, Macqueen GM, et al. Theory of mind deficits in patients with mild symptoms of major depressive disorder. Psychiatry Res 2013;210:672-4.

19. Michels R. Personality disorders in the depressed: seeing clearly through blue lenses. Am J Psychiatry 2010;167:487-8.

20. Hunot V, Moore TH, Caldwell D, et al. Mindfulness-based 'third wave' cognitive and behavioural therapies versus other psychological therapies for depression. Cochrane Database Syst Rev 2010(9).pii:CD008704

21. Jakobsen J, Gluud C, Kongerslev M, et al. 'Third wave' cognitive therapy versus mentalization-based therapy for major depressive disorder. A protocol for a randomised clinical trial. BMC Psychiatry 2012;12:232.

22. American Psychiatric Association. Diagnostic and Statistical Manual of Mental Disorders. Fourth update "text revison". DSM IV-TR. Washington, DC: American Psychiatric Association, 1994.

23. First MB, Spitzer RL, Gibbon M, et al. Structured clinical interview for DSM-IV TR axis I personality disorders, patient version (Danish translation). 2001

24. First MB, Spitzer RL, Gibbon M, et al. Structured clinical interview for DSM-IV TR axis II personality disorders, patient version (Danish translation). 1994

25. Bech A, Steer RA, Brown GK. Beck depression inventory-second edition: manual. San Antonio: The Psychological Corporation, 1996:4:561-71.

26. The National Health Service of Denmark. Guidance on treatment with antidepressants (in Danish). Ministry of Health, Denmark, 2000.

27. Jakobsen JC. 'Third wave' cognitive therapy. A treatment manual (in Danish). http://www.ctu.dk 2010.

28. Bokanowski T. Freud and Ferenczi: trauma and transference depression. Int J Psychoanal 1996;77:539.

29. Larsen KA. Mentalisation-based therapy. A treatment manual (in Danish). 2009. http://www.ctu.dk

30. Hamilton M. A rating scale for depression. J Neurol Neurosurg Psychiatry 1960;23:56-61.

31. Frank E, Prien RF, Jarrett RB, et al. Conceptualization and rationale for consensus definitions of terms in major depressive disorder. Remission, recovery, relapse, and recurrence. Arch Gen Psychiatry 1991;48:851-5

32. Derogatis L, Lipman RS, Covi L. SCL-90: an outpatient psychiatric rating scale. Psychopharmacol Bull 1973;9:13-28.

33. Bech P. Measuring the dimensions of psychological general well-being by the WHO-5. QoL News/ 2004;32:15-16.

34. Jakobsen JC, Gluud C, Winkel P, et al. The thresholds for statistical and clinical significance-a five-step procedure for evaluation of intervention effects in randomised clinical trials. BMC Med Res Methodol 2014;14:34

35. Brok J, Thorlund K, Gluud C, et al. Trial sequential analysis reveals insufficient information size and potentially false positive results in many meta-analysis. J Clin Epidemiol 2008;61:763-9.
36. DeMets DL, Lan KK. Interim analysis: the alpha spending function approach. Stat Med 1994;13:1341-56.

37. Thorlund K, Engstrøm J, Wetterslev J, et al. User manual for trial sequential analysis (TSA). Copenhagen Trial Unit, Centre for Clinical Intervention Research, Copenhagen, Denmark. 2011. p. 1-115. http://www.ctu.dk/tsa

38. Wetterslev J, Thorlund K, Brok J, et al. Trial sequential analysis may establish when firm evidence is reached in cumulative metaanalysis. J Clin Epidemiol 2008;61:64-75.

39. Jakobsen JC, Gluud C. The necessity of randomized clinical trials. Br J Med Res 2013;3:1453-68.

40. Schulz KF, Altman DG, Moher D. CONSORT 2010 statement: updated guidelines for reporting parallel group randomized trials. Ann Int Med 2010;152:726-32.

41. Switula D. Principles of good clinical practice (GCP) in clinical research. Sci Eng Ethics 2000;6:71-7.

42. Englev E, Petersen KP. ICH-GCP Guideline: quality assurance of clinical trials. Status and perspectives. Ugeskr Laeger 2003;165:1659-62.

43. Keus F, Wetterslev J, Gluud C, et al. Evidence at a glance: error matrix approach for overviewing available evidence. BMC Med Res Methodol 2010;10:90.

44. Higgins JPT, Green S. The Cochrane Handbook for Systematic Reviews of Interventions, Version 5.1.0. The Cochrane Collaboration. 2011. http://www.cochrane-handbook.org

45. Jakobsen JC, Hansen JL, Simonsen E, et al. The effect of interpersonal psychotherapy and other psychodynamic therapies versus 'treatment as usual' in patients with major depressive disorder. PLOS ONE 2011;6:e19044.

46. Jakobsen JC, Lindschou Hansen J, Storebø OJ, et al. The effects of cognitive therapy versus 'treatment as usual' in patients with major depressive disorder. PLoS One 2011;6:e22890

47. Helfand M, Berg A, Flum D, et al. Draft methodology report: ou questions, our decisions: standards for patient-centered outcomes research (Page 1-186). Patient-Centered Outcomes Research Institute. 2012; Published for Public Comment July 23 http://www. pcori.org/assets/Preliminary-Draft-Methodology-Report.pdf

48. Bassler D, Briel M, Montori VM, et al. Stopping randomized trials early for benefit and estimation of treatment effects: systematic review and meta-regression analysis. JAMA 2010;303:1180-7.

49. Guyatt GH, Briel M, Glasziou P, et al. Problems of stopping trials early. BMJ 2012;344:e3863.

50. Edwards BC, Lambert MJ, Moran PW, et al. A meta-analytic comparison of the Beck Depression Inventory and the Hamilton Rating Scale for Depression as measures of treatment outcome. Br J Clin Psychol 1984:23:93-9.

51. Lambert MJ, Hatch DR, Kingston MD, et al. Hamilton Rating Scales as measures of treatment outcome: a meta-analytic comparison. J Consult Clin Psychol 1986;54:54-9.

52. National Institute for Clinical Excellence. Depression: mangement of depression in primary and secondary care. London, England: National Institute for Clinical Excellence, 2004:640.

53. Jakobsen JC, Hansen JL, Simonsen S, et al. Effects of cognitive therapy versus interpersonal psychotherapy in patients with major depressive disorder: a systematic review of randomized clinical trials with meta-analyses and trial sequential analyses. Psychol Med 2011:42:1343-57.

54. Kirsch I, Deacon BJ, Huedo-Medina TB, et al. Initial severity and antidepressant benefits: a meta-analysis of data submitted to the Food and Drug Administration. PLoS Med 2008;5: e45.

55. Driessen E, Van HL, Don FJ, et al. The efficacy of cognitive-behavioral therapy and psychodynamic therapy in the outpatient treatment of major depression: a randomized clinical trial. Am J Psychiatry 2013;170:1041-50.

56. Chakraborty R, Chatterjee A. Predictors of suicide attempt among those with depression in an Indian sample: a brief report. Internet $J$ Ment Health 2006;4:2

57. Jakobsen J, Simonsen E, Rasmussen K, et al. Is the total score of the Hamilton Depression Rating Scale associated with suicide attempts or suicides? Br J Med Res 2013;3.

58. Bagby RM, Ryder AG, Schuller DR, et al.The Hamilton Depression Rating Scale: has the gold standard become a lead weight? Am J Psychiatry 2004;161:2163-77. 